\title{
Patient-reporting of side effects may provide an important source of information in clinical practice
}

\author{
Juliet M. Foster - Thys van der Molen • \\ Lolkje de Jong - van den Berg
}

Received: 8 May 2007 / Accepted: 8 June 2007 / Published online: 6 July 2007

(C) Springer-Verlag 2007

\begin{abstract}
Dear Editor,
The results of the recent study by Lampela et al. [1] showing a great disparity between adverse effects reported by physicians and those reported by patients is, in our opinion, not surprising. However, the direction of the disparity found in this study is intriguing. The side effects reported by 404 randomly selected elderly patients $(\geq 75$ years of age; mean drug use: 6.5 drugs) were compared with those identified by a physician. Overall physician-reporting of side effects (patients with one or more side effect $=24 \%$ ) was double that of patient-reporting $(11.4 \%)$. This is indeed surprising because it is well-documented that physicians often under-report [2] - and may even fail to discuss [3] side effects in clinical settings.
\end{abstract}

J. M. Foster $(\bowtie) \cdot$ T. van der Molen

Department of General Practice,

University Medical Center Groningen, University of Groningen,

Postbus 196, 9700 AD Groningen, The Netherlands

e-mail: J.M.Foster@med.umcg.nl

T. van der Molen

e-mail: T.van.der.Molen@med.umcg.nl

J. M. Foster $\cdot$ T. van der Molen

Department of General Practice and Primary Care,

University of Aberdeen,

Aberdeen, UK

L. de Jong - van den Berg

Department of Social Pharmacy, Pharmacoepidemiology

and Pharmacotherapy, University of Groningen,

Groningen, The Netherlands

e-mail: L.T.W.de.Jong-van.den.Berg@rug.nl
With more careful reading of the article a number of explanations for this finding emerge. Firstly, the study physician who assessed patients was highly trained and motivated to actively search for side effects, as the authors clearly state. In contrast, the patients had received no training about potential side effects, making it unlikely that unexpected side effects, such as cardiovascular or urinary problems, would be spontaneously reported. All data in the paper were from the intervention group, but data from the control group were not reported. This is unfortunate because control data would have provided important information about the rates of side effect reporting in patients who did not receive the intervention and from the physicians treating them. Secondly, participating patients reported their side effects in the context of a randomised intervention study, but patients taking part in trials may under-report their symptoms [4]. Thirdly, the method of assessing a side effect can be crucial to the level of patient-reporting achieved. The 'openquestion method' (for example: "have you had side effects?"), which is similar to that used in this study, may under-estimate patients' experiences of side effects while the 'rating-scale method' (in which the patient is provided with a questionnaire listing potential side effects) can drastically increase the patient's ability to report the side effects they experience [5].

In our view these interesting results from Lampela et al. should be regarded as a comparison of side effect reporting between expert-physician and naïve-patient using the openquestion method. Such results are unlikely to reflect what could be achieved with careful questioning of patients about side effects.

Finally, the authors conclude, perhaps correctly, that elderly persons tend to neglect drug side effects. The in- 
clusion of a comparison group of younger patients in this study would have been useful to support this supposition.

In conclusion, this study raises an important issue about disparity between physician- and patient-reporting of side effects. However, the results of this research represent optimal physician-reporting of side effects, and it is important that readers are aware that in everyday clinical practice there are often sub-optimal conditions for physician-reporting. Furthermore, patient-reporting of side effects is considered to be a useful source of information on side effects in pharmacovigilance [6] and clinical practice [7]. Thus, the authors' assertion that reliance on patient-reporting may lead to "many avoidable drug-related adverse effects (which) may be overlooked resulting in unnecessary distress to the patient" seems unfairly dismissive, considering that with the use of the appropriate methods (e.g. rating-scales), patient-reporting can be an important source of information about side effects in the context of real-life clinical practice.

\section{References}

1. Lampela P, Hartikainen S, Sulkava R, Huupponen R (2007) Adverse drug effects in elderly people - a disparity between clinical examination and adverse effects self-reported by the patient. Eur J Clin Pharmacol 63:509-515

2. Moride Y, Haramburu F, Requejo AA, Begaud B (1997) Underreporting of adverse drug reactions in general practice. Br J Clin Pharmacol 43:177-181

3. Britten N, Stevenson F, Gafaranga J, Barry C, Bradley C (2004) The expression of aversion to medicines in general practice consultations. Soc Sci Med 59:1495-1503

4. Rief W, Avorn J, Barsky AJ (2006) Medication-attributed adverse effects in placebo groups: implications for assessment of adverse effects. Arch Intern Med 166:155-160

5. Sheftell FD, Bigal ME, Feleppa M, Tepper SJ, Rapoport AM, Ciannella L (2004) Assessment of adverse events associated with triptans methods of assessment influence the results. Headache 44:978-982

6. van Grootheest AC, Passier JL, van Puijenbroek EP (2005) Direct reporting of side effects by the patient: favourable experience in the first year. Ned Tijdschr Geneeskd 149:529-533

7. Walker H, MacAulay K (2005) Assessment of the side effects of antipsychotic medication. Nurs Stand 19:41-46 